\title{
Acupuncture as an Adjunct to Standard Therapy for Pruritus in Patients with Atopic Dermatitis: A Patient- and Assessor-blinded, Randomized, Placebo-controlled Trial
}

\author{
Rona Maria R. Abad-Constantino, MD, ${ }^{1}$ Leah M. Caro-Chang, MD, ${ }^{1}$ Jolene Kristine G. Gatmaitan-Dumlao, MD, ${ }^{1}$ \\ Shahara Abalos-Babaran, MD, ${ }^{1}$ Philip Nino Tan-Gatue, $\mathrm{MD}^{2}$ and Belen L. Dofitas, $\mathrm{MD}^{1}$ \\ ${ }^{1}$ Department of Dermatology, Philippine General Hospital, University of the Philippines Manila \\ ${ }^{2}$ Department of Family and Community Medicine, Philippine General Hospital, University of the Philippines Manila
}

\begin{abstract}
Background. Pruritus can impair quality of life in patients with atopic dermatitis. There is evidence that acupuncture reduces pruritus and disease severity, and improves quality of life.

Objectives. This study aimed to determine the efficacy of acupuncture in reducing pruritus intensity, disease severity, and medication use, and improving quality of life.

Methods. This was a patient- and assessor-blinded, randomized, placebo-controlled trial. Patients diagnosed with atopic dermatitis underwent twice-weekly acupuncture for 12 weeks, with an 8-week follow-up period. Baseline and weekly assessment were done using standard data collection forms and validated assessment tools.

Results. Thirty patients were randomized and 28 patients were eligible for the efficacy analysis. There were no significant differences in the baseline demographic and clinical characteristics between the True Acupuncture group (TA) $(n=16)$ and Sham Acupuncture group (SA) $(n=12)$. Both groups showed reduction in mean itch intensity (visual analogue scale, VAS) $(p=0.024)$ but TA showed greater reduction $(p=0.009)$ that was sustained after end of treatment. There was also a reduction in medication use in both groups. The comparable efficacy of SA to TA is attributed to similar peripheral receptive fields and stimulation of cutaneous C-fibers which depletes the neurotransmitters mediating pruritus and results in tachyphylaxis. Mild adverse events, such as petechiae and erythema, were noted in both groups and resolved spontaneously.
\end{abstract}

Conclusion. Acupuncture is a promising adjunct treatment in atopic dermatitis with significant reduction in pruritus, disease severity and medication use and a trend towards improved quality of life. Studies with larger sample size and comparison to acupuncture points farther from the true acupuncture points are recommended.

Trial Registration. Food and Drug Administration Philippine Health Research Registry ID PHRR171012-001696

Key Words: Acupuncture, pruritus, atopic dermatitis, treatment adjunct

Paper presented in the Philippine Dermatological Society Research Forum 2019, on November 4, 2019, at the EDSA Shangri-La Hotel, Mandaluyong City, Philippines.

Corresponding author: Rona Maria R. Abad-Constantino, MD Department of Dermatology

Philippine General Hospital

University of the Philippines Manila

Taft Avenue, Ermita, Manila 1000, Philippines

Email: ronabad@gmail.com

\section{INTRODUCTION}

\section{Pruritus in Atopic Dermatitis}

Atopic dermatitis (AD) is a chronic, relapsing, inflammatory skin condition. It has an early onset but may develop in adulthood, with prevalence of $10 \%-30 \%$ in children and $2 \%-10 \%$ in adults. ${ }^{1,2}$

The diagnostic features of atopic dermatitis include typical morphology and distribution of skin lesions, chro- 
nically relapsing course, personal or family history of atopy, and generally dry skin. Pruritus is the hallmark of AD. ${ }^{2,3}$

\section{Pathophysiology of Pruritus in Atopic Dermatitis}

The mechanism of pruritus in $A D$ is not fully understood, but it is believed to be an interplay of endogenous and exogenous factors. Atopic individuals have a genetic impairment of epidermal barrier function leading to increased trans-epidermal water loss and reduced waterholding capacity of the stratum corneum. This leads to scaling, dryness, micro-fissuring, and itch. ${ }^{4}$ People with $\mathrm{AD}$ also have an inherent abnormality in the cutaneous immune response. Exogenous irritants, allergens, physical stimuli and emotional stress increase the propensity for skin irritation and pruritus. ${ }^{2}$

Pruritus is produced by different mediators (Table 1). Amines, proteases, neuropeptides, opioid peptides, neurotrophins, cytokines and inflammatory cells are all believed to play a role in the pathophysiology of pruritus in AD. ${ }^{5,6}$ These triggers and mediators stimulate itch receptors including free endings of thin, unmyelinated, slow-conducting C-fibers in the papillary dermis and epidermis. ${ }^{7}$ Once the sensation reaches the dorsal root ganglion and crosses the contralateral spinothalamic tract, it reaches different areas in the cortex.

A functional magnetic resonance imaging study demonstrated that aside from the motor cortex, involved in the scratch reflex, the anterior insula, striatum, and premotor and prefrontal cortices were also activated by allergeninduced pruritus. ${ }^{8}$ The putamen is implicated in motivation and habitual behavior underlying the urge to scratch. ${ }^{8,9} \mathrm{With}$ acupuncture, these areas have diminished activity, providing evidence for the central-acting effects of acupuncture. Moreover, neither antihistamines nor sham acupuncture reduced pruritus or altered brain response to evoked pruritus. ${ }^{8}$

With numerous mediators implicated in pruritus, antihistamines often fail to relieve pruritus in $\mathrm{AD}$ patients. ${ }^{6}$ A review of the efficacy of antihistamines in relieving pruritus has concluded that little evidence exists for the efficacy of $\mathrm{H} 1$ antihistamines in $\mathrm{AD} .{ }^{6,10,11}$ Thus, pruritus in $\mathrm{AD}$ is not primarily mediated by histamine, as there are many other mediators and nociceptive mechanisms involved in the pathophysiology of pruritus.

Table 1. Mediators of pruritus

\begin{tabular}{ll}
\multicolumn{1}{c}{ Class } & \multicolumn{1}{c}{ Specific type } \\
\hline Amines & Histamine 1, histamine 4, serotonin \\
\hline Proteinases & Trypsin, chymotrypsin \\
\hline Neuropeptides & $\begin{array}{l}\text { Substance P, vasoactive intestinal } \\
\text { peptide, Somatostatin }\end{array}$ \\
\hline Opioid peptides & Endorphins \\
\hline Neurotrophins & Nerve growth factor \\
\hline $\begin{array}{l}\text { Cytokines and } \\
\text { Inflammatory cells }\end{array}$ & IL-2, IL-31 \\
\hline
\end{tabular}

The impact of scratching on reduction of pruritus suggests that peripheral mechanisms of acupuncture may provide some relief. Insertion of an acupuncture needle into the tissue induces vasodilation around the needle through a stimulation of A-gamma or $\mathrm{C}$ fibers, secretion of mediators from inflammatory cells in the tissue, and depletion of neurotransmitters, resulting in tachyphylaxis. ${ }^{12}$

Although the pathways for pruritus and pain are distinct, $\mathrm{C}$-fibers are involved in both and it is believed that there is an overlap between the two. Patients with chronic pruritus have central sensitization for the symptom, wherein nociceptive stimuli of various modalities produce sensation of pruritus instead of pain. ${ }^{13}$

The exact pathogenesis of pruritus in $\mathrm{AD}$ remains unclear. Acupuncture interferes with central and peripheral pruritus transmission thereby contributing to its reduction. ${ }^{14}$ Figure 1 summarizes the proposed pathophysiologic mechanisms of pruritus.

\section{Management of Pruritus in Atopic Dermatitis}

Pruritus is an unpleasant sensation that is most disturbing to patients, resulting to impairment of quality of life. ${ }^{15}$ Atopic dermatitis has been called the "itch that rashes," which reflects the importance of controlling pruritus in its management. The combination of trigger elimination, topical therapy, systemic therapy, and other adjunctive treatment, are important in managing itch in AD. ${ }^{16}$ Topical therapy with emollients and corticosteroids is the first line of therapy, with the option of using calcineurin inhibitors and coal tar in severe disease. Systemic therapy are second-line options or first-line treatment in severe disease, which include oral antihistamines, cyclosporine $\mathrm{A}$, and oral corticosteroids.

Adjunctive modalities such as acupuncture may be used in addition to standard therapy. Acupuncture is the stimulation of specific points along the skin of the body. There are 51 acupuncture points listed by Xu Yihou that are used in the treatment of skin disorders (e.g., acne, psoriasis, dermatitis, and pruritus), and are classified according to function and indication. ${ }^{17,18}$ In traditional Chinese medicine (TCM), stimulation of specific points corrects imbalances in the flow of $q i$ through channels known as meridians. $Q i$ plays a central role and is considered the fundamental power underlying all the activities of nature and the vital life force of the human body. However, as this theory is not based upon generally accepted scientific knowledge, acupuncture remains controversial.

\section{Acupuncture Research: Itch Response in Patients with Atopic Eczema}

A literature search from the Cochrane Library and PubMed yielded six completed clinical trials and two systematic reviews. ${ }^{19-26}$

When comparing acupuncture therapy with placebo acupuncture (PA) and no acupuncture (NA), researchers were able to demonstrate the preventive effect of acupuncture 


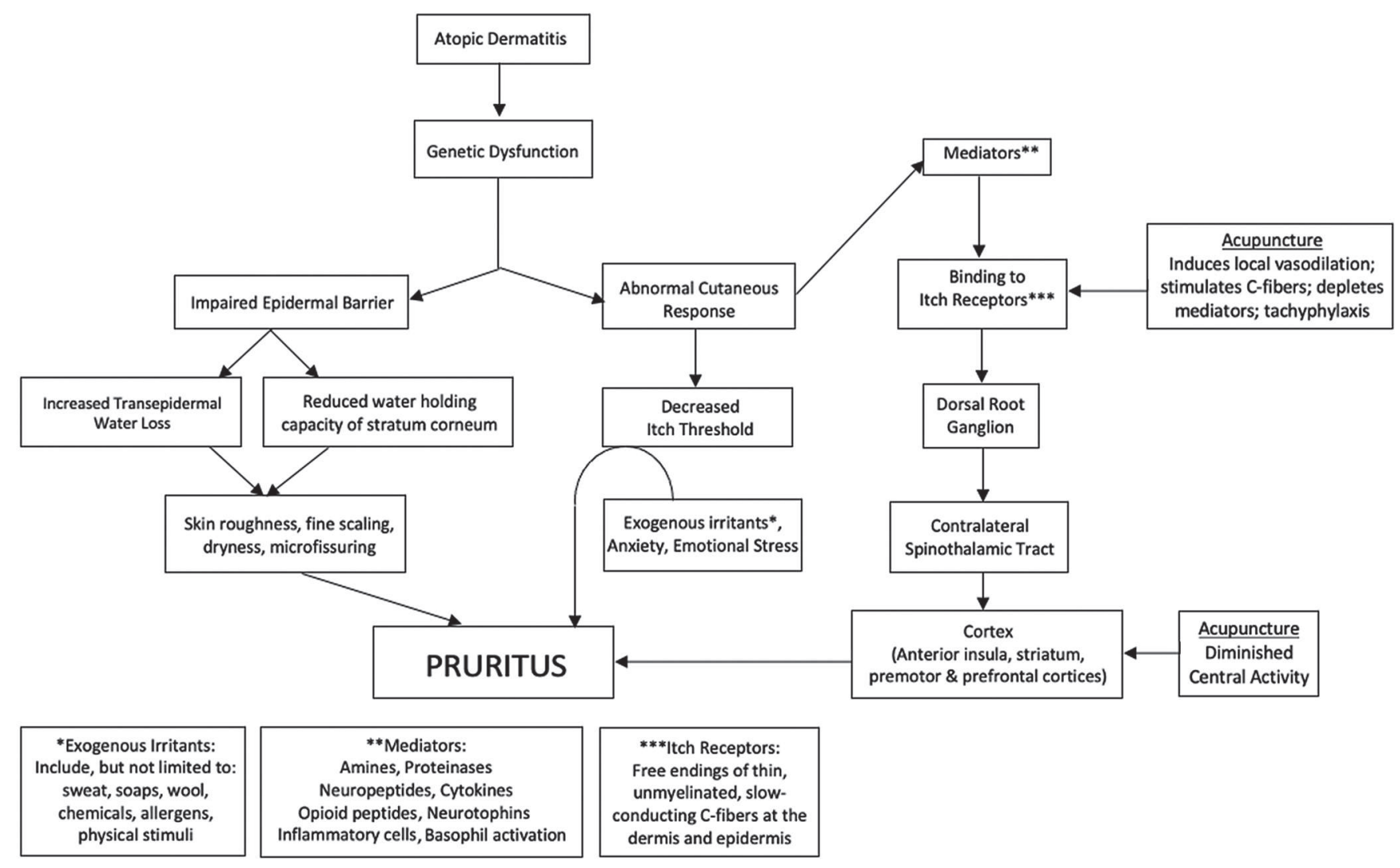

Figure 1. Pruritus Pathophysiology in Atopic Dermatitis. ${ }^{2,4-6,8-14}$

on histamine-induced itch, ${ }^{19}$ and that acupuncture lowered the itch intensity in $\mathrm{AD}$ patients given an allergen stimulus. ${ }^{20}$

A randomized controlled trial comparing acupuncture to PA and to cetirizine demonstrated that acupuncture was superior in controlling and reducing itch. ${ }^{21}$ This is congruent to earlier findings that neither itch sensation nor itchevoked brain response was altered following antihistamine or placebo acupuncture; and that greater itch reduction following acupuncture was associated with greater reduction in putamen response, ${ }^{8}$ a region implicated in motivation and habitual behavior underlying the urge to scratch.

In another clinical trial, patients were given combined therapy for 12 weeks, with acupuncture treatment twice a week and Chinese herbal medicine therapy 3 times daily. ${ }^{22}$ Assessment of eczema severity, itch severity, and quality of life was done every three weeks, as well as documentation of medication usage (topical corticosteroids and oral antihistamines) at each visit. At the end of the 12-week treatment period, all patients experienced a significant reduction in disease severity and itch severity, and improved quality of life. Medication use was also decreased by $39 \%$, with a significantly lower percentage of patients using topical corticosteroids and antihistamines during the last month of treatment compared to the 3-month prerecruitment period.

A recent systematic review with eight included RCTs further supports the efficacy of acupuncture in reducing itch intensity, and that it may be more effective than conventional medicine (topical corticosteroids and oral antihistamines) at improving disease severity and global symptoms for patients with $\mathrm{AD}^{23}$ Significant limitations of the review were high risk of bias due to the lack of blinding, poor methodological quality, and since half of the studies included were masteral theses.

Another systematic review by Hwang and Lio included two high-quality RCTs involving $\mathrm{AD}$ patients which showed contrasting results. ${ }^{24}$ The first study was able to demonstrate that acupuncture reduces itch intensity, disease severity, and improves quality of life, with significant differences between the acupuncture and sham groups. ${ }^{25}$ The second study compared acupuncture treatment and needleembedding treatment on their effects on disease severity, trans-epidermal water loss (TEWL), skin hydration, and quality of life. The researchers found a statistically significant improvement within both groups for all outcomes, but failed to see differences when comparing the two groups. ${ }^{26}$ In short, both acupuncture and needle-embedding treatment had positive effects on $\mathrm{AD}$ patients.

Different methodologies may explain these contrasting results. The first study used Park sham needles for their placebo group, while the other study used standard acupuncture needles in both groups. A Park sham needle is a validated device that appears similar to a real needle, but is blunt and 
free to slide within the handle so it telescopes into the handle instead of penetrating the skin. ${ }^{27}$ The contrasting findings from these studies highlight the importance of needle penetration in eliciting the beneficial effects of acupuncture.

Nevertheless, in all mentioned studies, itch intensity was highest for the no intervention group, showing that needle penetration, whether placebo or not, helps in reducing the sensation of itch. The complex pathophysiology of itch makes it difficult to pinpoint the exact mechanism that could explain these findings. But there is evidence that acupuncture may be effective in reducing the intensity of itch, and thus, may be a useful complementary treatment for pruritus in $\mathrm{AD}$.

\section{Significance of the Study}

Non-pharmacologic interventions for itch are rarely investigated, despite these having neither systemic nor serious side effects; thus, may be useful adjunctive therapy. Studies show that acupuncture reduces the intensity of itch, decreases severity, and improves quality of life. But to our knowledge, a randomized, controlled trial on the long-term effects of acupuncture on pruritus among patients with atopic dermatitis has not been conducted in the Philippine setting.

\section{METHODOLOGY}

This was a parallel group, patient- and assessor-blinded, randomized, placebo-controlled trial, with a 12 -week period of treatment and an 8-week follow-up period after the last treatment.

The study aimed to determine the long-term efficacy and safety of true acupuncture (TA) versus sham acupuncture (SA) on pruritus, disease severity, and quality of life among Filipino patients with atopic dermatitis.

\section{Outcome Measures}

The following outcomes were compared at baseline (week 0), weekly (weeks 1 to 12 during treatment period), and on follow-up (weeks 16, 20). A deviation of +2 days from a scheduled visit were allowed.

\section{Primary Outcomes}

The primary efficacy measure was the treatment response based on pruritus.

- the change in patient assessment of itch intensity (VAS): expressed as mean difference and \% mean difference and 95\% CI between VAS scores from baseline to another VAS measurement

\section{Secondary Outcomes}

- the change in the Scoring Atopic Dermatitis (SCORAD) index: mean difference and \% mean difference, 95\% CI

- the change in Dermatology Life Quality Index (DLQI) for participants aged 16 and above, and either Child Dermatology Life Quality Index (CDLQI) or Indeks $n g$ Kalidad ng Buhay Pangdermatolohiya ng mga Bata
(IKPaB) for participants aged 4 and below 16 years old: mean difference and $\%$ mean difference

- Global assessment (patient-reported)

- the amount of concurrent medication uses for AD

- the presence of adverse event during the treatment period and follow-up period

\section{Validated Tools for Outcome Measurement}

The following tools were used to measure the different outcomes:

1. Visual Analogue Scale (VAS) $)^{28-30}$ for itch intensity

2. Scoring Atopic Dermatitis (SCORAD) index ${ }^{31-33}$ for disease severity

3. Dermatology Life Quality Index (DLQI) ${ }^{34}$ in patients aged 17 to 65 years

4. Child Dermatology Life Quality Index (CDLQI) $)^{35}$ or Kalidad ng Bubay Pang-dermatolohiya ng mga Bata $(\mathrm{IKPaB})^{36}$ in patients aged 4 to 16 years

\section{VAS}

The VAS is a $10-\mathrm{cm}$ line on which patients indicate the intensity of pruritus by crossing the line at the point that corresponded to their pruritus severity, informed that the beginning of the scale indicates 'no itch' and the other end 'the worst itch they can imagine'.

\section{SCORAD}

The SCORAD index measures the extent, intensity, and subjective symptoms of $\mathrm{AD}$, combined to give a maximum possible score of 103. The score is interpreted as mild $(<25)$, moderate $(25-50)$, or severe $(>50)$.

\section{DLQI, CDLQI, IKPaB}

These Quality of Life (QoL) indexes measure how much a skin problem has affected the life of the patient over the previous seven days. They consist of ten items, and calculated by summing the score of each question resulting in a maximum of 30 and a minimum of 0 . The total score is interpreted as having 'no effect' (0-1), 'small effect' (2$5)$, 'moderate effect' (6-10), 'very large effect' (11-20), or 'extremely large effect' (21-30) on the patient's life.

A higher VAS score means more intense itch, while a higher SCORAD score indicates a more severe disease condition. The higher the quality-of-life score, the more effect the skin condition has on the patient's life, and therefore, a poorer quality of life.

\section{Setting}

This study was conducted in the Philippine General Hospital, a tertiary care hospital in Metro Manila, from July 2016 to November 2018. Baseline assessments for each patient was done at the dermatology clinic by the investigators before directing the patient to the treatment facility (Traditional and Alternative Medicine Clinic) for the acupuncture session. 
Acupuncture for Atopic Dermatitis

\section{Sample size}

The main indicators for the sample size were the frequency of outcome measurement periods and the expected difference between the two treatment groups. Each patient was measured 15 times, and the difference was set at 5 . With power of $81 \%, 5 \%$ level of significance and a standard deviation of 2 , a repeated measures design with 1 between factor and 1 within factor has 2 groups with 11 participants each for a total of 22 patients. Allowing for 20\% drop out rate, the minimum sample size requirement was increased to 28 .

\section{Recruitment and selection of participants}

Convenience sampling was employed and participants were recruited through advertisements posted in bulletin boards around the hospital. Patients recruited had to be of sound mind, between 4 and 65 years of age, and with atopic dermatitis (AD). Restriction to this age range was made because the assessment tool for patient perceived quality of life have only been validated for this age group. Each volunteer was screened by the investigators to ensure diagnosis of $\mathrm{AD}$, according to the criteria developed by Williams et al. ${ }^{3}$

The exclusion criteria were: (1) treatment with phototherapy, oral or intravenous immunosuppressive agents or corticosteroids in the preceding four weeks before recruitment; (2) treatment with acupuncture and/or Chinese herbal medicine (CHM) in the previous three months; (3) pregnancy; and (4) severe physical or mental illness, particularly those with blood dyscrasia and severe anemia.

Written informed consent of eligible adult patients, parents of patients, and written assent were secured from patients aged 12-17. Participants were then randomly assigned to their treatment groups.

Simple randomization (1:1) was done using a computergenerated list of random numbers. The treatment arm was coded as either 1 or 2 by a third party who was not directly involved in the clinical trial. The third party was contacted for the treatment allocation after a patient was enrolled in the study. The patient's numerical code was written in the case record form (CRF) prior to scheduling for acupuncture. Each enrolled patient was allocated to either the acupuncture or the sham treatment group but was not informed about which treatment he/she was receiving. Only the acupuncturist knew which codes referred to acupuncture or sham treatment.

Entries in the CRFs were encoded after each visit by a research assistant. All CRFs were collected at the end of the study period.

The outcome assessors, data collator and biostatistician were blinded to the treatment allocation. The investigators screened, performed data collection and outcome assessments. The treatment groups were only revealed after the statistical analysis was completed.

\section{Study procedure}

\section{Procedure}

Each patient received either true acupuncture (TA) or sham acupuncture (SA) twice weekly, with at least a oneday interval between the treatment sessions, lasting for 30 minutes. Overall, each patient was expected to complete 24 sessions by the end of the 12 -week treatment period.

Patients continued to receive conventional treatment and were regularly seen by their physicians every week during the 12-week treatment period. Outcome measures were assessed, and any adverse events or worsening of symptoms were noted. They were advised to use mild moisturizing soap and emollients regularly, and topical corticosteroids and oral antihistamines as needed. The amount of topical corticosteroids and oral antihistamines used for the past month were recorded at each visit, using a standard recording sheet that was given to each patient. They were also allowed to continue any medication they were taking for concomitant diseases.

\section{Acupuncture Points}

Selection of acupuncture points for the TA group was based on previous studies on acupuncture and upon consultation with a licensed acupuncturist co-investigator. ${ }^{17}$ The licensed acupuncturist determined the non-acupuncture points as well. He performed the acupuncture procedures for all patients. He is a certified medical acupuncturist by the Philippine Academy of Acupuncture, Inc and has been practicing since 2005 .

For the true acupuncture points, two points from the upper extremities and two points from the lower extremities were selected for balance. For this study, the following points were used: Hegu (LI-4), Quichi (LI-11), Fengshi (GB-31), and Zusanli (St-36) (Figure 2, Figure 4). These were manually stimulated for 5-10 seconds with a depth of one cun with muscle twitch ( $Q i$ sensation) as the sought response. These points are empiric points for itch and are believed to be involved in immunomodulation. ${ }^{17}$

For sham points, needles were inserted (depth of one cun) in acupuncture points not specific for pruritus (PC-6, LI-10, ST-38, and SP-6) (Figure 2, Figure 4) and without manual stimulation and muscle twitch. A tracking form was given to the acupuncturist to ensure proper type and frequency of intervention being given to each patient. The acupuncturist signed the form after each acupuncture session.

\section{Materials and Resources}

Each patient was alloted 8 sterile one-cun needles with diameter of $0.25 \mathrm{~mm}$ (Huanqiu brand) per acupuncture session (Figures 3 and 4). A total of 192 needles were provided for each patient for the 24 acupuncture sessions.

Figure 5 summarizes the study procedure flow chart. 

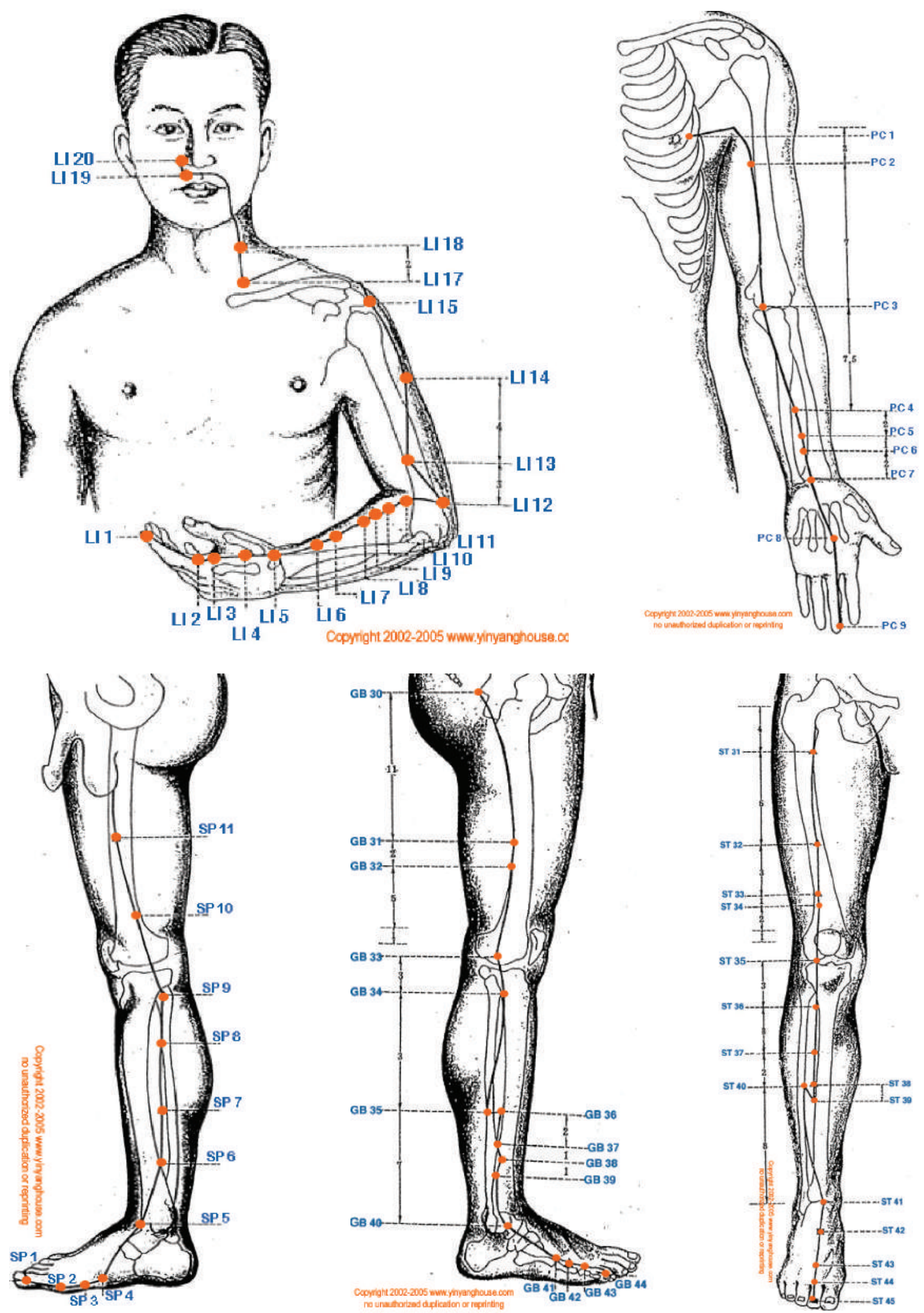

Figure 2. Body diagram of acupuncture points used in the study. Boxed in blue used in True Acupuncture. Boxed in red used in Sham Acupuncture. 

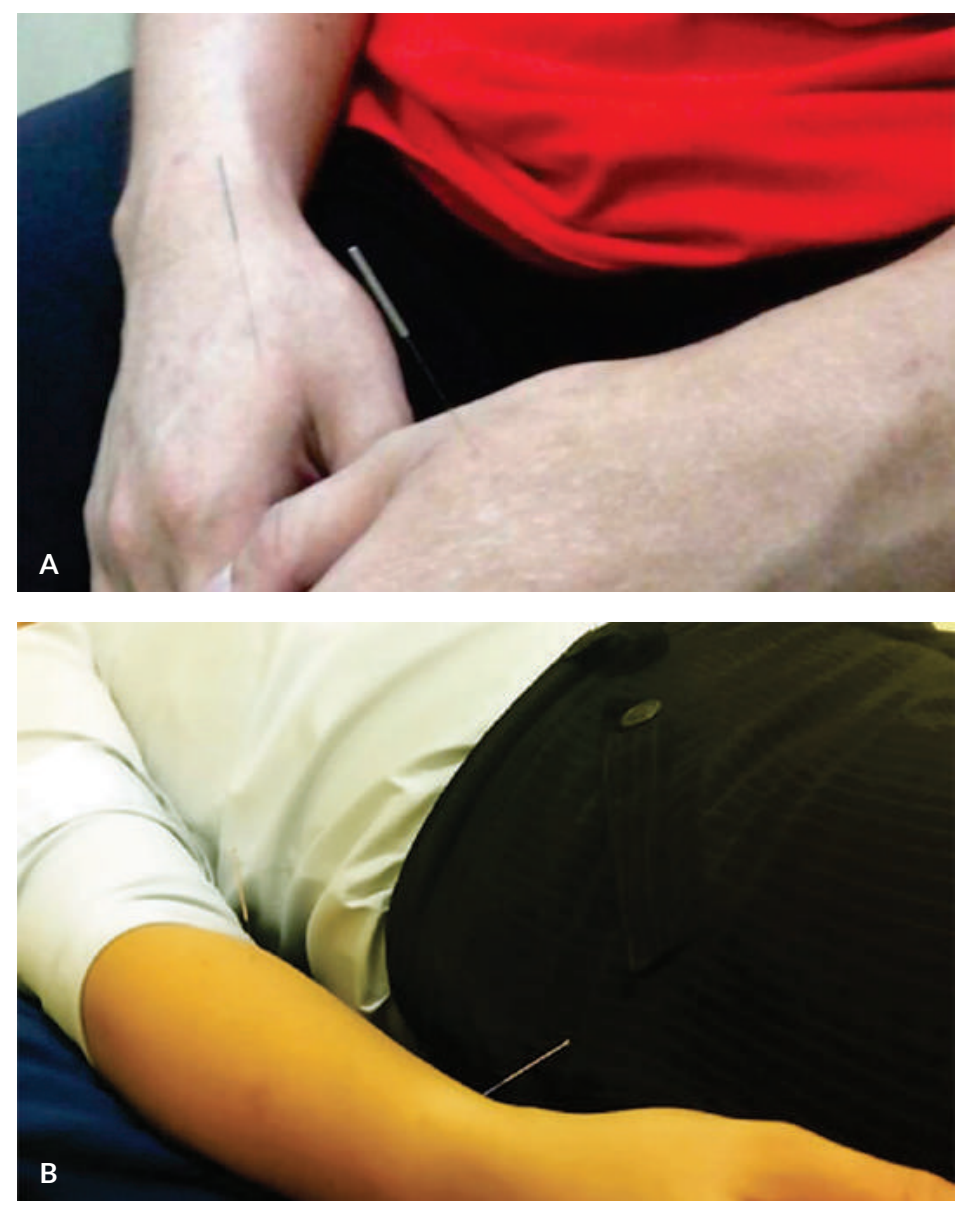

Figure 3. Close-up view of needles during acupuncture treatment.

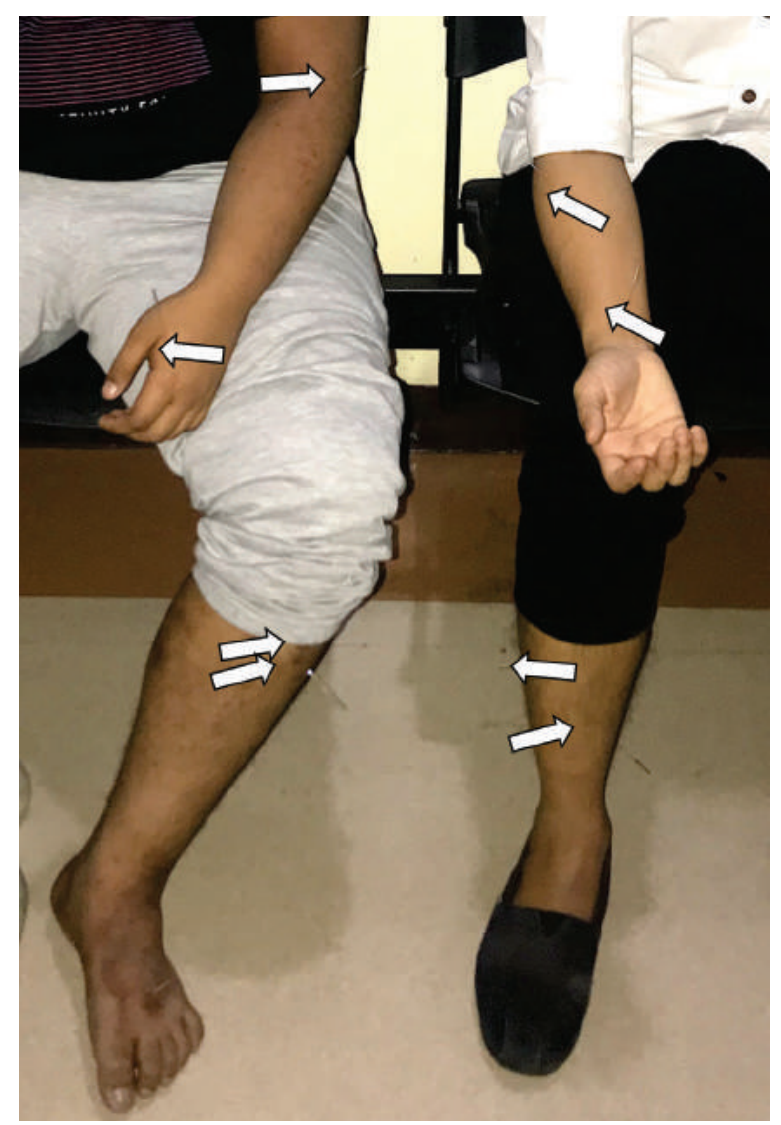

Figure 4. Patient on the left received treatment 1 (true). Patient on the right received treatment 2 (sham). Note location needles (white arrows).

\section{Data Collection}

The standard questionnaires for assessment of itch intensity, disease severity, and quality of life were administered by the investigators on the first visit and in the subsequent visits. All questionnaires and forms were kept by the primary investigator and filed according to patient control number.

Each patient's baseline medication uses for the last one to three months prior to recruitment were recorded during the first visit. For the subsequent months included in the study duration, a form for recording medication use was given to each patient. Patients were instructed to record the amount of medication use from the date they received the form until the date of the next reassessment.

\section{Evaluation of Blinding}

At the end of the study, each patient was asked whether they thought they received true acupuncture or sham acupuncture using a separate questionnaire. Patients were allowed to answer "not sure."

\section{Handling of non-compliers and drop-outs}

Patients who were unable to follow up and receive treatments as scheduled were considered non-compliant.
They were encouraged by the investigators to adhere to the protocol and received reminders as well. Dropouts were patients who discontinued their treatment without informing the investigators. Investigators attempted to contact the dropouts to find out the reasons. Non-compliers were included in the modified intention-to-treat analysis. Dropouts were included in the sensitivity analysis.

\section{Data Management and Statistical Analysis}

A standard CRF was used to collate the data for each patient. This form included demographics and comorbidities. Identifiers such as birthdate and contact information were asked to ensure correct patient identification at follow-up. Data were collated and encoded in Microsoft Excel 2007 by a hired research assistant (RA). Each patient's data set from every assessment period was turned over for compilation.

The acupuncturist gave the list of patients (with their control numbers) in 'treatment 1' and in 'treatment 2' to the data collator. The RA then encoded each patient's data in two separate Microsoft Excel files, to either 'treatment 1' and 'treatment 2 .'

The collated data were given to the biostatistician for analysis. All patients randomized were included in the 


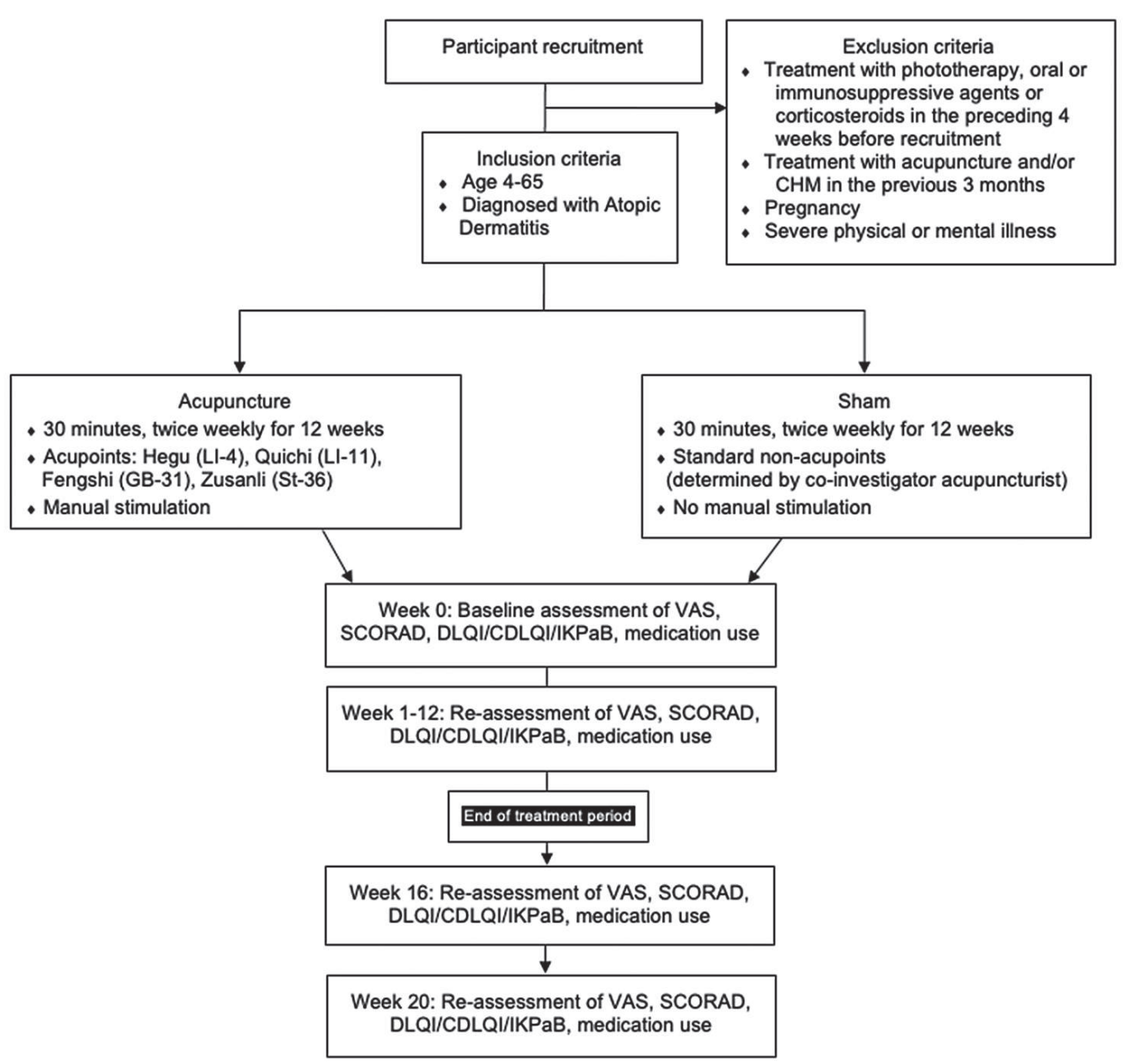

Figure 5. Study procedure flow chart.

modified intention-to-treat (ITT) analysis provided there was at least one follow-up outcome recorded. The last observation was 'moved forward' and considered the end-oftreatment observation in the analysis.

Enrolled patients who dropped out without any observation recorded beyond the baseline measures were included in a sensitivity analysis to estimate the effect of these dropouts on the conclusion.

Comparison of baseline characteristics between the intervention and control groups was done using independent t-test for quantitative variables, and Fisher's exact test for qualitative variables. Comparisons of continuous variables (SCORAD, VAS, health-related quality of life) between the two groups across different time points were performed using the Repeated Measures Analysis of Variance (ANOVA). All comparisons made were two-tailed, and $p$-values $\leq 0.05$ were considered to be statistically significant. Intercooled STATA 12 was used in the analysis. Microsoft Office Excel was utilized to generate graphs for presentation of results.

Dichotomous outcomes such as treatment success or failure was analyzed for relative risk (RR), relative risk reduction (RRR), absolute risk reduction (ARR), number-needed-to treat (NNT), and their respective 95\% confidence intervals. An online EBM calculator was used.

\section{Criteria for Treatment Success and Treatment Failure}

Treatment success: All of the following conditions should be present:

1. SCORAD: No change in disease severity, or improvement defined as at least a 4-point decrease from baseline, at the end of the treatment period

2. VAS: Decrease in itch severity, with a 3-5\% difference from baseline in mean VAS at the end of the treatment period

3. DLQI/CDLQI/IKPaB: Improvement defined as at least a 3-point score decrease from baseline at the end of the treatment period

4. Global assessment of patient: Improved or cleared

Treatment failure: Any of the following conditions are present:

1. SCORAD: Worsening of disease severity at the end of the treatment period, with at least a 4-point increase from baseline 


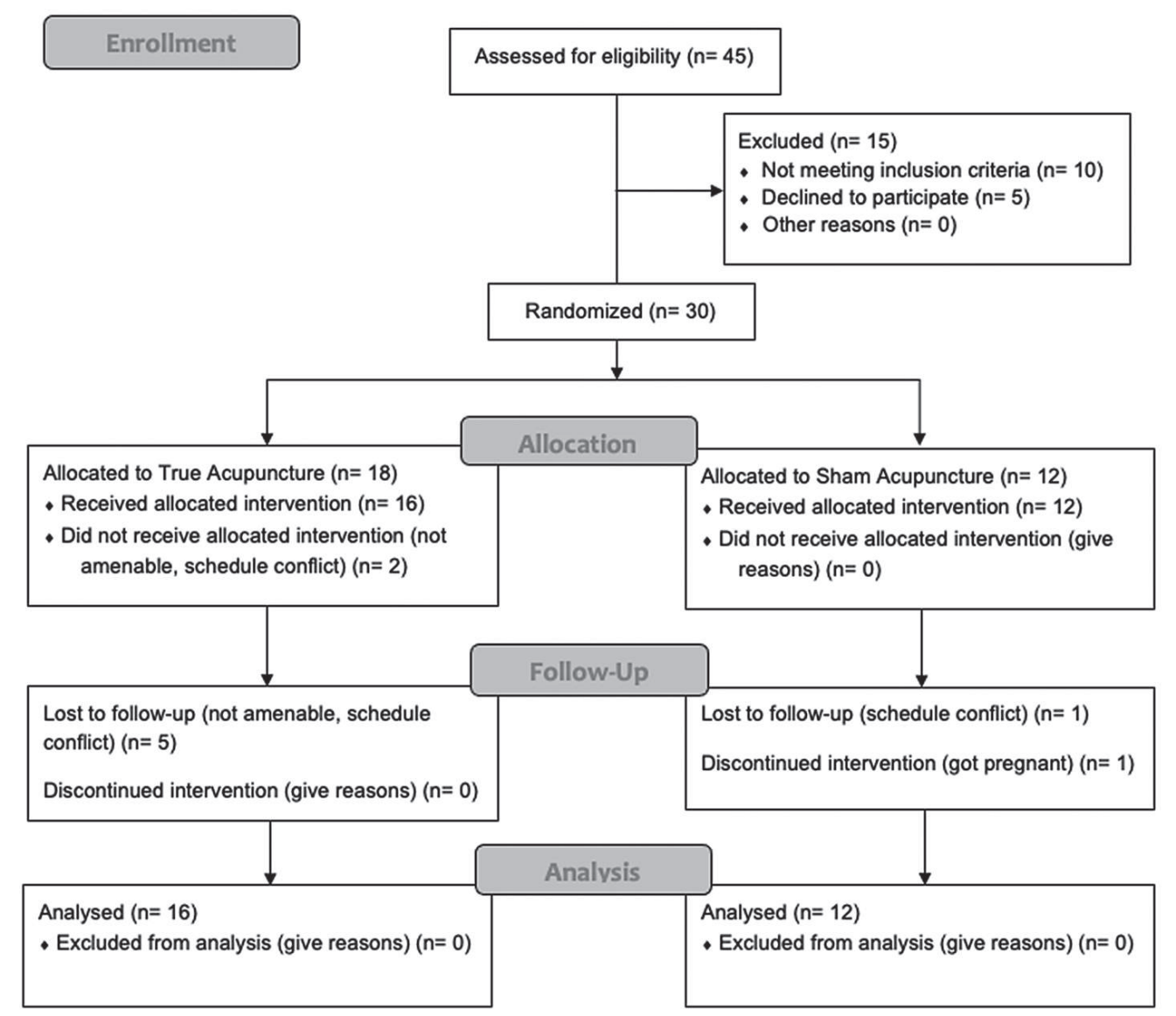

Figure 6. Flowchart of the enrolment, allocation, and analyzed sample.

2. VAS: $<3 \%$ difference from baseline in mean VAS at the end of the treatment period

3. DLQI/CDLQI/IKPaB: Worsening of quality of life, with a 3-point score increase from baseline at the end of the treatment period

4. Global assessment of patient: No change from baseline or worsened

\section{RESULTS}

Forty-five patients were screened for eligibility. Thirty patients (17 men, 13 women) with atopic dermatitis with ages ranging from 7-53 years were recruited for the study. Two patients, both randomized to treatment 1 (true acupuncture), did not receive the treatment after enrolment, and were considered drop outs from the study. The first patient was not amenable to the procedure and the second patient had schedule conflicts.

Twenty-eight patients were eligible and included in the efficacy analysis (Figure 6). Seven were considered noncompliant $(\mathrm{TA}=5, \mathrm{SA}=2)$ and did not complete the study. One patient became pregnant in the middle of the study, and the remainder had schedule conflicts.

\section{Patient Baseline Characteristics}

There was no significant differences in terms of age, sex, and co-morbidities between the true acupuncture (TA) group and sham acupuncture (SA) group (Table 2). Interpretation of SCORAD and DLQI showed that both groups had moderate atopic dermatitis which had very large effects on the patients' quality of life.

\section{Pruritus}

\section{Mean Itch Intensity (VAS)}

The mean itch intensity showed a decreasing trend in both groups (Figure 7). Repeated measures ANOVA showed that the reduction in VAS across time for both groups was significant $(\mathrm{p}<0.001)$. Moreover, the difference between the groups was also significant $(\mathrm{p}=0.02)$.

Across time, each treatment group showed a significant mean reduction of VAS from baseline $(\mathrm{p}=0.024)$. The greater mean reduction in pruritus in TA (5.24 points) compared to SA (3.76 points) was statistically significant $(\mathrm{p}=0.009)$ (Table 3). The TA group had a larger reduction in terms of percentage mean difference from baseline (76.93\%) compared to sham treatment $(58.34 \%)$ although this difference was not significant across time between groups (18.59\%, 95\% CI -17.83 to 55.01$)$. 
Table 2. Baseline characteristics

\begin{tabular}{|c|c|c|c|}
\hline & $\begin{array}{c}\text { True } \\
\text { Acupuncture } \\
(\mathrm{N}=16) \mathrm{n}(\%)\end{array}$ & $\begin{array}{c}\text { Sham } \\
\text { Acupuncture } \\
(\mathrm{N}=12) \mathrm{n}(\%)\end{array}$ & p-value \\
\hline Age (years) & $27.0 \pm 1.0$ & $31.5 \pm 11.7$ & 0.37 \\
\hline \multicolumn{4}{|l|}{ Sex } \\
\hline Male & $8(50)$ & $8(67)$ & \multirow{2}{*}{0.46} \\
\hline Female & $8(50)$ & $4(33)$ & \\
\hline \multicolumn{4}{|l|}{ Comorbidities } \\
\hline Asthma & $6(38)$ & $3(25)$ & 0.69 \\
\hline Allergic Rhinitis & $2(13)$ & $3(25)$ & 0.62 \\
\hline Others & $2(13)$ & $0(0)$ & 0.49 \\
\hline \multicolumn{4}{|l|}{ Medication Use } \\
\hline Topical corticosteroids & $9(56)$ & $5(42)$ & 0.70 \\
\hline Oral antihistamine & $6(38)$ & $1(8)$ & 0.18 \\
\hline Emollient Use & $9(56)$ & $5(42)$ & 0.70 \\
\hline VAS & $7.1 \pm 2.5$ & $5.6 \pm 3.3$ & 0.18 \\
\hline SCORAD & $41.0 \pm 14.1$ & $36.7 \pm 15.4$ & 0.45 \\
\hline DLQI/CDLQI & $11.7 \pm 7.0$ & $11.5 \pm 6.7$ & 0.94 \\
\hline
\end{tabular}

VAS, Visual analogue scale; SCORAD, SCORing Atopic Dermatitis; DLQI, Dermatology Life Quality Index; CDLQI, Children's Dermatology Life Quality Index

\section{Global Assessment of Itch (Patient-reported)}

At week 12, ten patients (63\%) in TA reported improvement of pruritus, whereas two patients (13\%) noted worsening of pruritus and four patients (25\%) reported no change. In SA, seven (58\%) patients reported improvement, one patient (8\%) noted worsening of pruritus, and four patients (25\%) reported no change in pruritus. These differences were not statistically significant $(\mathrm{p}=1)$ (Table 4).

At the end of the follow-up period (week 20), the TA group had nine (56\%) patients who still reported improvement, five patients who reported worsening of their pruritus (31\%), while two (13\%) reported no change. In the sham group, seven (58\%) patients reported no change and only four $(33 \%)$ reported improvement in pruritus. One patient $(8 \%)$ reported worsening of pruritus. These differences in patient-reported assessment of itch were significant $(\mathrm{p}=0.043)$ (Table 4).

\section{Scoring of Atopic Dermatitis (SCORAD)}

The SCORAD showed a decreasing trend (Figure 8) across the treatment period for both groups indicating improvement in atopic dermatitis, from a baseline of "moderate" severity to "mild" severity by week 12 . The TA group had slighty lower SCORAD scores compared to SA at week 12 (8.36 vs 9.19) and week 20 (11.29 vs. 13.5), with greater reduction noted in TA as well. Repeated measures
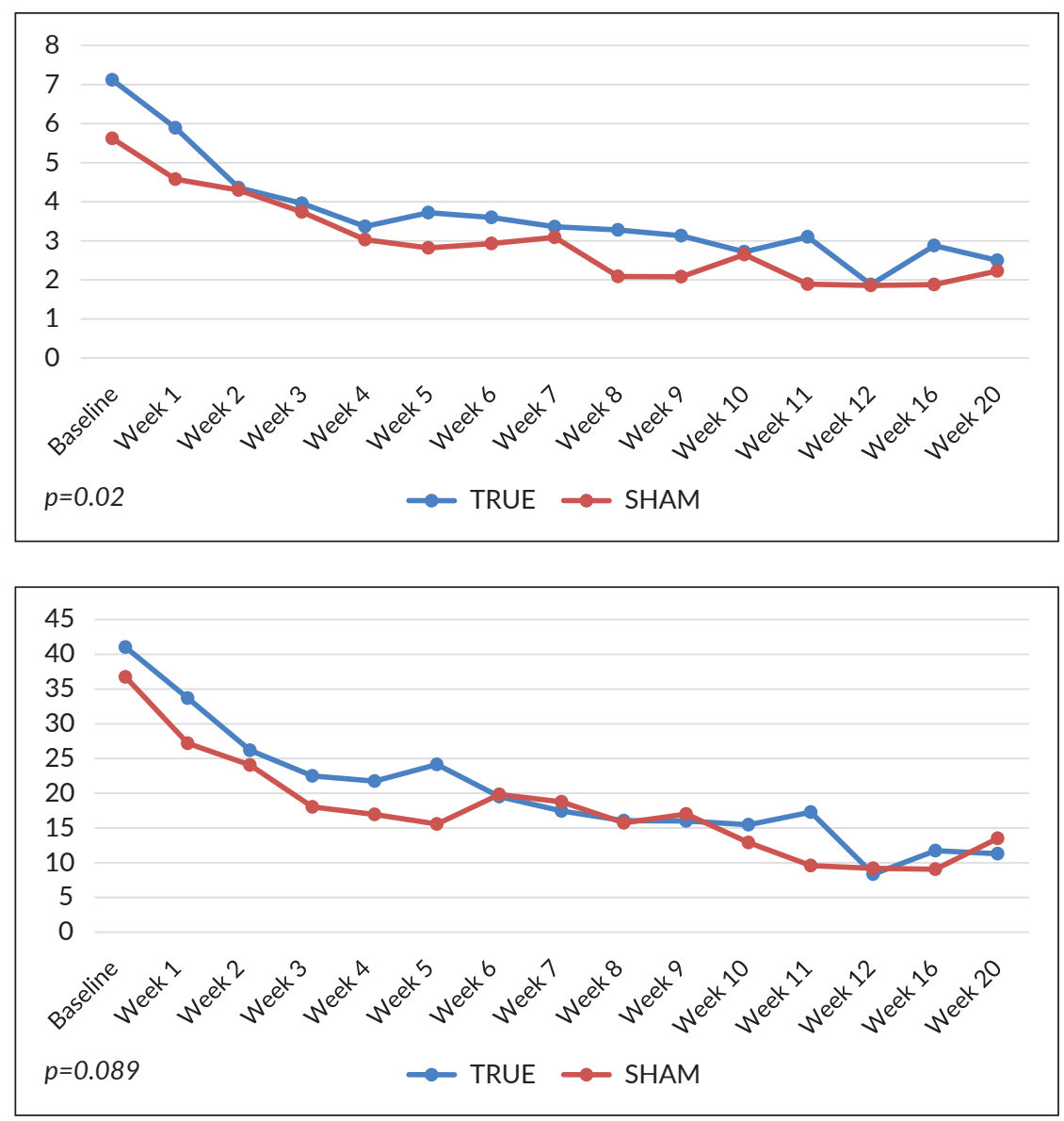

Figure 7. Comparison of mean itch intensity (VAS) from baseline and across the 12 weeks of treatment and followup at week 16 and week 20. 
Table 3. Outcomes at the end of the treatment period (week 12) and follow-up period (week 20)

\begin{tabular}{|c|c|c|c|}
\hline Outcome & $\begin{array}{l}\text { True Acupuncture } \\
(\mathrm{N}=16)\end{array}$ & $\begin{array}{c}\text { Sham Acupuncture } \\
(\mathrm{N}=12)\end{array}$ & Difference $(95 \% \mathrm{Cl})$ \\
\hline \multicolumn{4}{|l|}{ From baseline to week 12} \\
\hline Mean change in pruritus (VAS) & $5.24 \pm 2.94$ & $3.76 \pm 3.35$ & $1.48(-0.97$ to 3.93$)$ \\
\hline Mean change in disease severity (SCORAD) & $32.68 \pm 14.89$ & $27.55 \pm 16.50$ & $5.13(-7.11$ to 17.36$)$ \\
\hline Mean change in quality of life (DLQI/CDLQI) & $8.13 \pm 6.37$ & $7.17 \pm 7.25$ & $0.96(-4.34$ to 6.26$)$ \\
\hline \multicolumn{4}{|l|}{ From baseline to week 20} \\
\hline Mean change in pruritus (VAS) & $4.62 \pm 3.28$ & $3.38 \pm 4.06$ & $1.23(-1.62$ to 4.09$)$ \\
\hline Mean change in disease severity (SCORAD) & $29.74 \pm 19.32$ & $23.24 \pm 17.80$ & 6.5 (-8.17 to 21.18$)$ \\
\hline Mean change in quality of life (DLQI/CDLQI) & $7.5 \pm 6.55$ & $6.58 \pm 7.68$ & $0.92(-4.62$ to 6.45$)$ \\
\hline
\end{tabular}

Table 4. Global assessment of Itch (patient-reported) at the end of the treatment period (week 12) and at the end of the follow-up period (week 20)

\begin{tabular}{llrlll} 
& $\begin{array}{c}\text { Treatment } \\
\text { Group }\end{array}$ & Improved & Worsened & No change & p-value \\
At week 12 & True & $10(63)$ & $2(13)$ & $4(25)$ & \multirow{2}{*}{1} \\
& Sham & $7(58)$ & $1(8)$ & $4(33)$ & \\
\hline At week 20 & True & $9(56)$ & $5(31)$ & $2(13)$ & \multirow{2}{*}{0.04} \\
& Sham & $4(33)$ & $1(8)$ & $7(58)$ & \\
\hline
\end{tabular}

ANOVA showed that the mean reduction in SCORAD across time for both groups was significant $(p<0.001)$, but the differences between these groups was not significant $(\mathrm{p}=0.089)$.

\section{Quality of Life}

The DLQI and CDLQI scores also showed trends towards improvement in both groups (Figure 9). From a baseline of "moderate effect on QoL," both treatment groups improved to "small effect on QoL" by week 12 and week 20. Repeated measures ANOVA showed that the reduction in DLQI/CDLQI across time for both groups was significant $(p<0.001)$ but the differences between the groups was not significant $(p=0.34)$. The QoL scores at the end of treatment (week 12) and follow-up (week 20) were slightly lower in TA $(3.56 \pm 4.37$ and $4.19 \pm 5.99$, respectively) than $\mathrm{SA}(4.33 \pm 5.10$ and $4.92 \pm 6.63$, respectively) indicating a greater improvement in quality of life in TA.

\section{Assessment of Treatment Effect}

Treatment effects at week 12 were not significantly different between groups but acupuncture at the correct points showed a trend towards reducing the risk of treatment failure (i.e., risk of worsening of either pruritus, SCORAD, DLQI, or global assessment) by 25\% (RRR 25\%) compared to sham treatment (Table 5) and this holds true even when sensitivity analysis was carried out (RRR 14.3\%) (Table 6).

\section{Medication Use}

There was a reduction in topical corticosteroid use (Figure 10) use and oral antihistamine intake (Figure 11) for both groups throughout the treatment period and follow-up.

Topical corticosteroid use for TA was initially used by nine patients (56\%) with no patients using topical corticosteroids at the end of the treatment period (week 12) and only one patient (6\%) applied topical corticosteroids during the end of follow-up (week 20). For SA, only one patient $(8 \%)$ used it at week 12 and continued to use it up to week 20 compared to five patients (42\%) at baseline.

For intake of oral antihistamine drugs, none of the patients in TA group took antihistamines at week 12 up to week 20. As for SA, there was no oral antihistamine intake at week 12 and only one patient (8\%) took antihistamines at week 20 .

Table 5. Assessment of treatment effect (Endpoint: Treatment failure at Week 12)

\begin{tabular}{|c|c|c|c|c|c|c|}
\hline $\begin{array}{l}\text { Treatment } \\
\text { Group }\end{array}$ & $\begin{array}{l}\text { Treatment Failure } \\
\qquad(n=14)\end{array}$ & $\begin{array}{l}\text { Treatment Success } \\
\qquad(\mathrm{n}=14)\end{array}$ & $\operatorname{RR}(95 \% \mathrm{Cl})$ & $\operatorname{RRR}(95 \% \mathrm{Cl})$ & $\operatorname{ARR}(95 \% \mathrm{Cl})$ & NNT (95\% Cl) \\
\hline True & 7 & 9 & \multirow{2}{*}{$0.75[0.36$ to 1.56$]$} & \multirow{2}{*}{$25 \%[-0.561$ to 0.64$]$} & \multirow{2}{*}{$14.6 \%[-0.205$ to 0.45$]$} & \multirow{2}{*}{$7[-4.45$ to 1.94$]$} \\
\hline Sham & 7 & 5 & & & & \\
\hline
\end{tabular}

Table 6. Assessment of treatment effect (Sensitivity Analysis)

\begin{tabular}{|c|c|c|c|c|c|c|}
\hline $\begin{array}{l}\text { Treatment } \\
\text { Group }\end{array}$ & $\begin{array}{l}\text { Treatment Failure } \\
\qquad(n=16)\end{array}$ & $\begin{array}{l}\text { Treatment Success } \\
\qquad(n=14)\end{array}$ & $\mathrm{RR}(95 \% \mathrm{Cl})$ & $\operatorname{RRR}(95 \% \mathrm{Cl})$ & $\operatorname{ARR}(95 \% \mathrm{Cl})$ & NNT $(95 \% \mathrm{Cl})$ \\
\hline True & 9 & 9 & \multirow{2}{*}{$0.86[0.44$ to 1.67$]$} & \multirow{2}{*}{$14.3 \%[-0.667$ to 0.559$]$} & \multirow{2}{*}{$8.3 \%[-0.25$ to 0.39$]$} & \multirow{2}{*}{$12[-4$ to 3$]$} \\
\hline Sham & 7 & 5 & & & & \\
\hline
\end{tabular}




\section{Adverse Events}

Five patients $(\mathrm{TA}=3, \mathrm{SA}=2)$ experienced petechiae at the sites of acupuncture but these resolved with application of cold compress in one patient and spontaneously resolved after 24 hour in others (Grade $1 \mathrm{AE}$ ).

One patient (SA group) reported persistent erythema three days post-acupuncture. Complete blood count and bleeding parameters done were all within normal limit. The erythema resolved spontaneously after five days. (Grade $1 \mathrm{AE})$
Another patient (SA group) developed furunculosis on the forehead on week 11 of treatment. The furunculosis did not develop on the area of the acupuncture points. The patient was prescribed by his primary physician with oral antibiotics (doxycycline) with persistence. This patient has a long-standing history of recurrent appearance of multiple erythematous pustules on the face previously treated with amoxicillin-clavulanic acid and clindamycin. Cultures were requested which yielded MRSA, resistant to clindamycin. He was given rifampicin with resolution of lesions (Grade $2, \mathrm{AE}$, unexpected and not related to acupuncture).
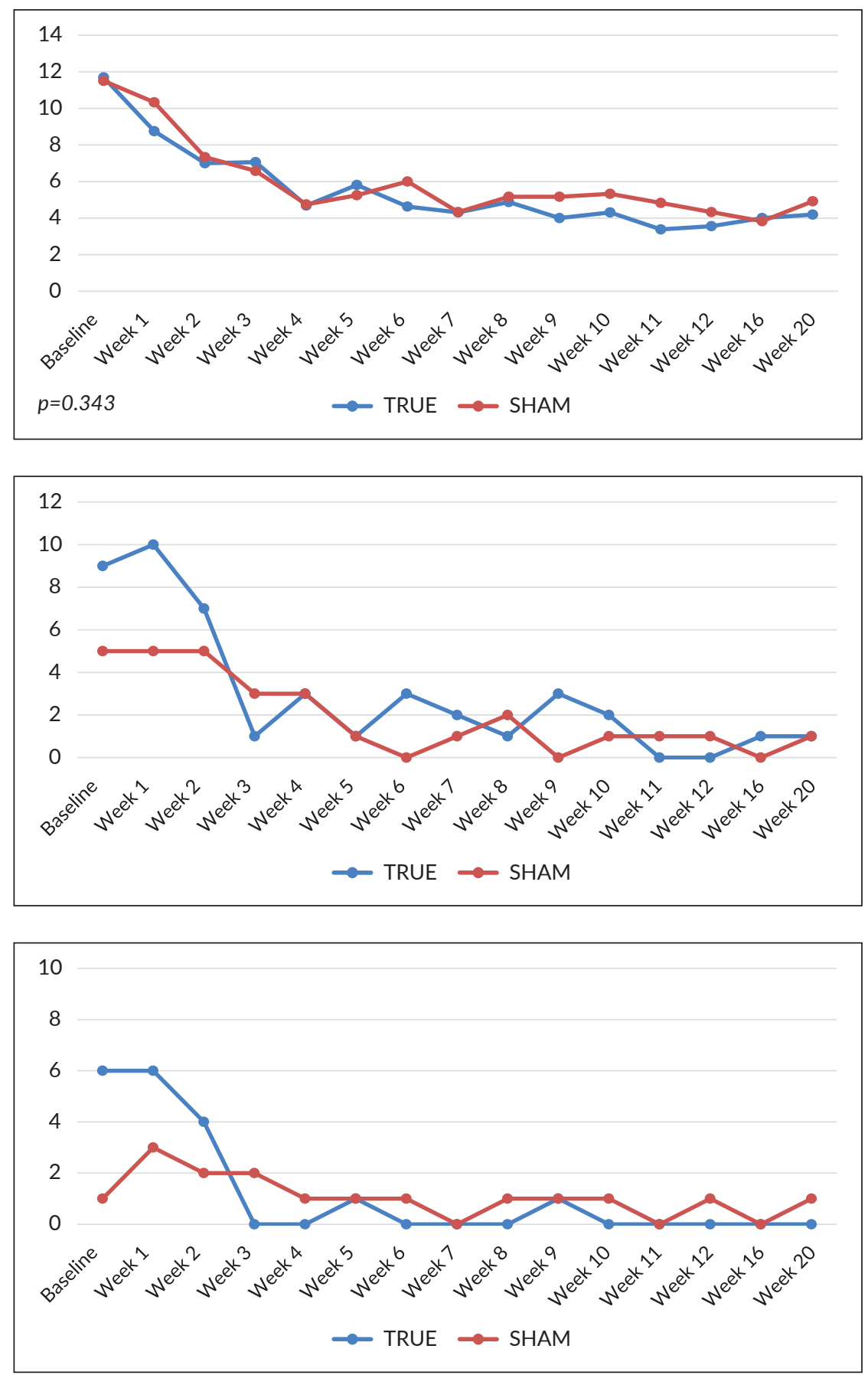

Figure 9. Comparison of patient perceived quality of life (DLQI/CDLQI) from baseline and across the 12 weeks of treatment and follow-up at week 16 and week 20.

Figure 10. Comparison of topical corticosteroid use from baseline and across the 12 weeks of treatment and follow-up at week 16 and week 20.

Figure 11. Comparison of oral antihistamine intake from baseline and across the 12 weeks of treatment and follow-up at week 16 and week 20. 
Table 7. Evaluation of Blinding

\begin{tabular}{crlcc} 
Treatment Group & True & Sham & Unsure & No answer \\
True Acupuncture & $12(75)$ & 0 & 0 & $4(26)$ \\
Sham Acupuncture & $3(25)$ & $4(33)$ & $1(8)$ & $4(33)$ \\
\hline
\end{tabular}

\section{Efficacy of Blinding}

Majority of the patients in TA, 12 out of $16(75 \%)$, answered that they underwent true acupuncture (Table 7). The remainder had no answer. For the patients in SA, three (25\%) thought they had true acupuncture, four (33\%) thought they received sham acupuncture, one (8\%) was unsure, and the remaining four (33\%) had no answer.

\section{DISCUSSION}

Previous studies have reported significant reduction of itch with use of acupuncture. Acupuncture acts both centrally and peripherally, to have anti-allergic, anti-inflammatory, and antipruritic effects. ${ }^{25}$ It was found to control itch sensation by reduction of type 1 hypersensitivity reactions and basophil activation, ${ }^{11}$ decreasing serum IgE levels, ${ }^{25}$ and decreasing nerve fiber density. ${ }^{14}$ It was also found to decrease TEWL and increase skin hydration, ${ }^{26}$ which greatly benefits atopic individuals as they have a genetic impairment of epidermal barrier function.

In this study exploring acupuncture as an adjunct to atopic dermatitis, there was greater reduction in itch intensity (VAS) in the group given acupuncture at the points noted to relieve pruritus - LI-4, LI-11, GB-31 and ST-36. However, a similar trend was noted in the sham group who underwent acupuncture at points not specific for pruritus - PC-6, LI10, ST-38, and SP-6 and without manual stimulation. A clinical trial by Pfab et al. was similar to our study in that both placebo and acupuncture led to pruritus reduction, but acupuncture at correct points led to greater reduction. ${ }^{20}$ In this study, placebo acupuncture entailed needles inserted in the same dermatomes as those of the intervention group but not from the classic meridian system. There are several mechanisms that will provide an understanding of the results of this study.

\section{Cutaneous Stimulation of C-fibers}

Scratching the skin and the consequential reduction of pruritus suggests that cutaneous stimulation of $\mathrm{C}$-fibers in the epidermis and dermis, such as needle insertion, induces vasodilation and stimulation that depletes the neurotransmitters mediating pruritus and results in tachyphylaxis. ${ }^{12}$ It has also been demonstrated that after 10 sessions of acupuncture in a non-specific acupoint for itch, there is a reduction in nerve fiber density and therefore less sensory enervation on the area. ${ }^{14}$ Hence, in the group who underwent sham acupuncture, the mere act of needle insertion provided decrease in pruritus, albeit weaker, than those who underwent acupuncture at points identified through modulations of neuronal activity described earlier. In fact, according to previous studies, any active intervention on pruritus, whether placebo or not, helps reduce the sensation. ${ }^{11,19-21}$

\section{Peripheral Receptive Fields}

A peripheral receptive field $(\mathrm{RF})$ is defined as a skin area stimulated, and which influences the activity of a spinal sensory neuron. ${ }^{37} \mathrm{RF}$ s may span the area around an acupuncture point; hence, stimulation of areas adjacent to acupuncture points would evoke therapeutic effects. Reviewing the diagram of the areas stimulated, LI-10 (stimulated in SA) was very close to LI-11 (stimulated in TA) and ST-38 (SA) was also rather close to (ST-36). SP-6 (SA) is close to other pre-identified points for pruritus - SP-10. Despite needling at a non-target site, a control procedure within the same myotome provides similar effects. Moreover, a RF may occur in both acupuncture and non-acupuncture points. Stimulation of these RFs still send signals that converge in the central nervous system and translate to pruritus reduction. ${ }^{37}$

\section{Stimulus intensification}

It was demonstrated that patients with chronic pruritus have central sensitization for the symptom. ${ }^{13}$ This central sensitization is also coupled with a peripheral sensitization whereby light stimulation of the skin or acupuncture without manipulation may produce an effect as strong as acupuncture at true points..$^{38}$ In relation to the concept of receptive fields, central sensitization also leads the expansion of RFs producing effects similar or equivalent to the precise acupuncture point when needles are inserted within such area. ${ }^{37}$ For those who do not have central sensitization a control procedure with needling in a nearby myotome produces effects when needling within the affected myotome. ${ }^{38}$

\section{Acupoints and meridians}

In TCM, acupoints are located in areas where the $q i$ of meridians is streaming. The needle sensation is believed to be an essential element of acupuncture representing the needle's effect in controlling the flow of energy $(q i)$ in the body. ${ }^{27} \mathrm{By}$ stimulating these points, the natural flow of $q i$ is restored when these are impaired in pathologic conditions. ${ }^{17,37}$ In examining the acupuncture points of the supposed sham group, stimulation of points belonging to the same meridian were noted - large intestine (LI-10 in SA vs. LI-4 and -11 in TA) and stomach (ST-38 in SA vs. ST-36 in TA). Moreover, SP-6, stimulated in SA, is a selected point for dry and lichenified skin with desquamation and itching. ${ }^{17} \mathrm{It}$ is also used for the treatment of eczema thereby providing improvements in terms of skin dryness, lichenification and desquamation. ${ }^{39}$

The stimulation of $\mathrm{C}$-fibers and receptive fields as well as the stream of $q i$ in proper acupuncture points and meridians provide vital points in understanding the reduction in pruritus in the sham group. Topical corticosteroid use and oral antihistamine intake were markedly decreased in both 
groups at the end of the treatment period and follow-up, with both the patients and physicians deeming them not necessary for their disease states at the evaluation.

Patient-reported assessment of pruritus were similar in both groups with majority reporting improvement at the end of the treatment period. On follow-up, however, more patients reported a continued state improvement in TA whereas more patients noted no change in their pruritus two months after the last session in SA. It can be hypothesized that the stimulation of the correct acupuncture points in TA provide improvement that lasts longer compared to the points in SA.

For disease severity (SCORAD) and quality of life (DLQI and CDLQI), improvements were noted in both acupuncture and sham groups. There was greater reduction in scores in the acupuncture group (though not statistically significant) and this was even greater during the last week of follow-up. This may be attributed to the stimulation of correct acupuncture points that do not only provide immediate relief of pruritus but one that is sustained even after treatment cessation.

\section{Study Limitations}

The following limitations may have affected the study results. First is the absence of a group that did not receive acupuncture. Non-inclusion of such a group was deliberate to maintain blinding among the participants. However, it was not foreseen that placement of sham acupoints would have significant results as well, and having a group that did not receive acupuncture would have yielded stronger evidence for acupuncture's efficacy. In relation to this, the lack of access to Park sham needles such as those used by Kang et al. ${ }^{25}$ is also a limitation. Park sham needles are blunt, nonpenetrating acupuncture needles that have been validated in studies. ${ }^{27}$ In this study, there was needle penetration in both acupuncture and sham groups, and this action may be responsible for the beneficial effects.

In addition, based on the evaluation of blinding, there is a possibility that the participants had an inkling whether or not they received acupuncture or sham. This may be due to the set up in the Traditional and Alternative Medicine Clinic where the acupuncture sessions were done. The sessions were held in one room, at times simultaneously, and the participants could have shared their experiences with one another.

Lastly, because the study was designed for acupuncture to be an adjunct treatment, the improvements seen in both acupuncture and sham groups may be due to the use of conventional medications for AD. Note that the use of topical and oral medications were allowed in this study, as withholding these medications from patients who need them may be problematic due to ethical reasons. With the study design, it is difficult to ensure that the improvements were not from the medications alone. The research findings are promising and exciting, but should still be interpreted with caution.

\section{CONCLUSION AND RECOMMENDATIONS}

This study concludes that acupuncture, whether on established points for pruritus or otherwise, provides potential reduction of pruritus with improvement in VAS, SCORAD and DLQI after 12 weeks of treatment. Moreover, acupuncture at the correct points provided greater reduction that is sustained two months after the last acupuncture session with positive effects on patients' quality of life. There was also a reduction in topical corticosteroid use and oral antihistamine intake. Acupuncture at LI4, LI-11, GB-31 and ST-36 for 12 weeks is a promising treatment adjunct to atopic dermatitis.

To achieve a sham acupuncture treatment, the acupuncture points should be farther from these correct points (i.e. different myotome, meridian, as well as not a pre-defined acupuncture point). Alternatively, future studies may use blunted Park sham needles that will allow treatment blinding, but not needle penetration. A better clinic set up would also help in patient blinding. Setting up the treatment room with dividers will prevent patients from seeing one another and minimize patient communication while receiving acupuncture therapy. Furthermore, individualized treatment for the patients, with additional perilesional or lesional points, are worth exploring as well.

This study provides some evidence that acupuncture may be included in the treatment armamentarium of atopic dermatitis since it showed a trend to benefit and it was well tolerated even by pediatric patients as young as ten years old. Further randomized controlled trials with a larger sample size are needed to further validate acupuncture as an effective and safe adjunct to the control of pruritus among pediatric and adult atopic dermatitis patients.

\section{Statement of Authorship}

All authors participated in the data collection and analysis and approved the final version submitted.

\section{Author Disclosure}

All authors declared no conflicts of interest.

\section{Funding Source}

This study was funded by the Philippine Institute of Traditional and Alternative Health Care (PITAHC).

\section{REFERENCES}

1. Asher M, Montefort S, Bjorksten B. Worldwide time trends in the prevalence of symptoms of asthma, allergic rhinoconjunctivitis, and eczema in childhood: ISAAC Phases One and Three repeat multicountry cross-sectional surveys. Lancet. 2006; 368:733-4.

2. Bieber T, Bussman C. Atopic dermatitis. In Jorrizo, J, Schaffer J, editors. Bolognia Dermatology. 3rd ed. Philadelphia: Elsevier Saunders; 2012: 203-17.

3. Williams H, Burney P, Pembroke A, Hay R. The U.K. Working Party's Diagnostic Criteria for Atopic Dermatitis. III. Derivation of 
a minimum set of discriminators for atopic dermatitis. Br J Dermatol. 1994; 131:406-16.

4. Del Rosso J. Repair and maintenance of the epidermal barrier in patients diagnosed with atopic dermatitis. J Clin Aesthet Dermatol. 2011; 4(6):45-55.

5. Charlesworth E, Beltrani V. Pruritic dermatoses: overview of etiology and therapy. Am J Med. 2002; 113(9A):25S-33S.

6. Stander S, Luger T. Itch in atopic dermatitis - pathophysiology and treatment. Acta Dermatovenerol Croat. 2010; 18(4):289-96.

7. Darsow U, Pfab F, Valet M, Huss-Marp J, Behrendt H, Ring J, et al. Pruritus and atopic dermatitis. Clin Rev Allerg Immuno. 2011; 41:237-44.

8. Napadow V, Li A, Loggia M, Kim J, Schalock P, Lerner E, et al. The brain circuitry mediating antipruritic effects of acupuncture. Cereb Cortex. 2014;24:873-882.

9. Vierow V, Hukuoka M, Ikoma A, Dorfler A, Handwerker H, Forster C. Cerebral representation of the relief of itch by scratching. J Neurophysiol. 2009; 102(6):3216-24.

10. Klein P, Clark R. An evidence-based review of the efficacy of antihistamines in relieving pruritus in atopic dermatitis. Arch Dermatol. 1999; 135:1522-5.

11. Pfab F, Athanasiadis G, Huss-Marp J, Fuqin J, Heuser B, Cifuentes L, et al. Effect of acupuncture on allergen-induced basapohil activation in patients with atopic eczema: a pilot trial. J Altern Complement Med. 2011; 17(4):309-14.

12. Carlsson C, Wallengren J. Therapeutic and experimental therapeutic studies on acupuncture and itch: review of literature. JEADV;2010; 24:1013-6.

13. Ikoma A, Fartasch M, Heyer G, Miyachi Y, Handwerker H, Schmelz M. Painful stimuli evoke itch in patients with chronic pruritus: central sensitization for itch. In: Neurology. 2004: 62;212-7.

14. Carlsson C, Sundler F, Wallengren J. Cutaneous innervation before and after one treatment period of acupuncture. Br J Dermatol. 2006; 155:970-6

15. Lundberg L, Johannesson M, Silverdahl M, Hermansson C, Lindberg $\mathrm{M}$. Health-related quality of life in patients with psoriasis and atopic dermatitis measured with SF-36, DLQI and a subjective measure of disease activity. Acta Derm Venereol. 2000; 80:430-4.

16. Hong J, Buddenkotte J, Berger T, Steinhoff M. Management of itch in atopic dermatitis. Semin Cutan Med Surg. 2011; 30(2):71-86.

17. Xu Y. Acupuncture and Moxibustion. Dermatology in Traditional Chinese Medicine. China: Donica Publishing; 2004:56-60.

18. Tan E, Millington G, Levell N. Acupuncture in dermatology: an historical perspective. Int J Dermatol. 2009; 48(6):648-52.

19. Pfab F, Hammes M, Backer M, Huss-Marp J, Athanasiadis G, Tölle T, et al. Preventive effect of acupuncture on histamine-induced itch: a blinded, randomized, placebo-controlled, crossover trial. J Allergy Clin Immunol. 2005; 116(6):1386-8.

20. Pfab F, Huss-Marp J, Gatti A, Fuqin J, Athanasiadis G, Irnich D, et al. Influence of acupuncture on type I hypersensitivity itch and the wheal and flare response in adults with atopic eczema - a blinded, randomized, placebo-controlled, crossover trial. Allergy. 2010; 65(7): 903-10.

21. Pfab F, Kirchner M, Huss-Marp J, Schuster T, Schalock P, Fuqin $\mathrm{J}$, et al. Acupunture compared to oral antihistamine for type I hypersensitivity itch and skin response in adults with atopic dermatitis - a patient and examiner blinded, randomized, placebo-controlled, crossover trial. Allergy. 2012; 67(4):566-73.
22. Salameh F, Perla D, Solomon M, Gamus D, Barzilai A, Greenberger $\mathrm{S}$, et al. The effectiveness of combined chinese herbal medicine and acupuncture in the treatment of atopic dermatitis. J Altern Complement Med. 2008; 14(8):1043-8.

23. Jiao R, Yang Z, Wang Y, Zhou J, Zeng Y, Liu Z. The effectiveness and safety of acupuncture for patients with atopic eczema: A systematic review and meta-analysis. Acupunct Med. 2020; 38(1):3-14.

24. Hwang J, Lio P. Acupuncture in dermatology: An update to a systematic review. The J Altern Complement Med. 2020; 0(0):1-12.

25. Kang S, Yu-Kang K, Yeom M, Lee H, Jang H, Park H, et al. Acupuncture improves symptoms in patients with mild-to-moderate atopic dermatitis: A randomized, sham-controlled preliminary trial. Complement Ther Med. 2018; 41:90-8.

26. Lee H, Park S. Prelimenary comparison of the efficacy and safety of needle-embedding therapy with acupuncture for atopic dermatitis patients.Evid-Based Compl AltMed.2019.doi:10.1155/2019/6937942

27. Park J, White A, Stevinson C, Ernst E, James M. Validating a new non-penetrating sham acupuncture device: two randomised controlled trials. Acupunct Med. 2002 Dec; 20(4):168-74.

28. Reich A, Heisig M, Phan N, Taneda K, Takamori K, Takeuchi S, et al. Visual analogue scale: evaluation of the instrument for the assessment of pruritus. Acta Derm Venereol. 2012; 92:497-501.

29. Reich A, Szepietowski J. Pruritus intensity assessment: challenge for clinicians. Expert Rev Dermatol. 2013; 8(3):291-9.

30. Reich A, Halupczok J, Ramus M, Stander S, Szepietowski J. New data on the validation of VAS and NRS in pruritus assessment: minimal clinically important difference and itch frequency measurement. Acta Derm Venereol. 2011; 91:636.

31. Rehal B, Armstrong A. Health outcome measures in atopic dermatitis: A systematic review of trends in disease severity and quality-of-life instruments 1985-2010. PLoS ONE. 2011; 6(4):17520.

32. Schmitt J, Langan S, Deckert S, Svensson A, Kobyletzki L, Thomas $\mathrm{K}$, et al. Harmonising Outcome Measures for Atopic Dermatitis (HOME) Initiative. (2013). Assessment of clinical signs of atopic dermatitis: a systematic review and recommendation. J Allergy Clin Immunol. 2013; 132(6):1337-47.

33. European Task Force on Atopic Dermatitis. Severity scoring of atopic dermatitis: the SCORAD index (consensus report of the European task force on atopic dermatitis). Dermatology. 1993; 186:23-31.

34. Finlay A, Khan G. Dermatology Life Quality Index (DLQI) - a simple practical measure for routine clinical use. Clin Exp Dermatol. 1994; 19(3):210-6.

35. Lewis-Jones M, Finlay A. The children's dermatology life quality index (CDLQI): initial validation and practical use. Br J Dermatol. 1995; 132(6):942-9.

36. Gonzales-Carait P, Genuino R, Dofitas B, Reyes K. Validation of Indeks ng Kalidad ng Buhay Pangdermatolohiya ng mga Bata (IKPaB): a Filipino translation of the Children's Dermatology Life Quality Index (CDLQI) text version. Acta Med Philipp. 2014; 54(3):270-7.

37. Quiroz-Gonzalez S, Torres-Castillo S, Lopez-Gomez R, Estrada I. Acupuncture points and their relationship with multreceptive fields of neurons. J Acupunct Meridian. 2017; 10(2):81-9.

38. Lund I, Naslund J, Lundeberg T. Minimal acupuncture is not a valid placebo control in radomised controlled trials of acupuncture: a physiologist's perspective. Chin Med. 2009; 4(1):1-9.

39. Shen D, Wu X, Wang N. Acupuncture and Moxibustion. Manual of Dermatology in Chinese Medicine. USA: Eastland Press, Inc.; 1995:39-40. 